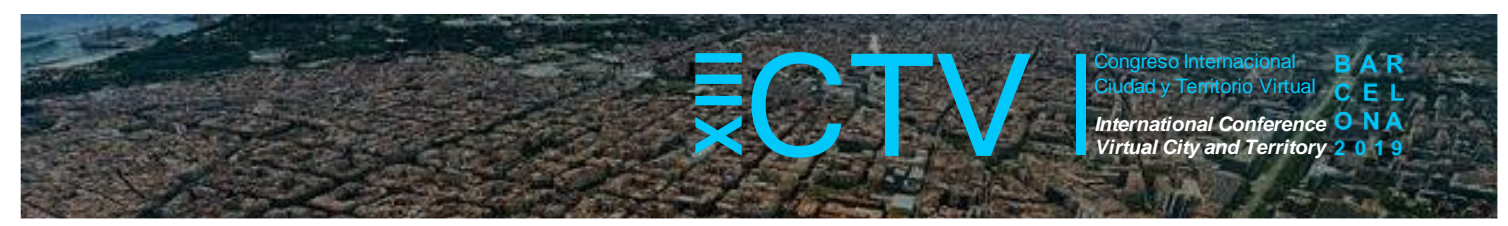

\title{
LAS CIUDADES MEDIAS EN JALISCO, UNA ALTERNATIVA DE DESARROLLO SOSTENIBLE, A TRAVÉS DEL PROCESO DE METROPOLIZACIÓN PARA LA CONSOLIDACIÓN DE LAS REGIONES
}

\author{
Orendain Verduzco, Tomas Eduardo1*; Michel Parra, J. Guadalupe²; Montañez Valdez, Oziel \\ Dante ${ }^{3}$; Martínez Ibarra, José Alejandro ${ }^{4}$, Gutiérrez Cedillo, Aurora Berenice ${ }^{5}$
}

Remisión inicial: 2019-05-31; Remisión definitiva: 2019-10-17; Publicación: 2019-12-21

Citación: Orendain Verduzco, T. E. et al. (2019). Las Ciudades Medias en Jalisco, una alternativa de Desarrollo Sostenible, a través del proceso de Metropolización para la consolidación de las Regiones. En XIII CTV 2019 Proceedings: XIII International Conference on Virtual City and Territory: "Challenges and paradigms of the contemporary city": UPC, Barcelona, October 2-4, 2019. Barcelona: CPSV, 2019, p. 8496. E-ISSN 2604-6512. DOI http://dx.doi.org/10.5821/ctv.8496

\begin{abstract}
Resumen
Las Ciudades Medias en proceso de consolidación como áreas Metropolitanas en Jalisco, generan expectativas positivas como una alternativa de "Desarrollo Sustentable", y descentralización del área Metropolitana de Guadalajara, Jalisco, México. Este estudio se centrará en dos zonas y/o áreas metropolitanas siendo la zonas metropolitana de Ocotlán, en la Región Ciénega del Estado de Jalisco, México y área metropolitana del Sur de Jalisco, en la Región Sur del Estado, además de la implementación de instrumentos como la elaboración y/o actualización del programa metropolitano a través del Ordenamiento Territorial en aras de buscar la equidad para el Desarrollo Urbano Sustentable que busque impactar de una manera más equilibrada en sus regiones.
\end{abstract}

Esta investigación tiene un enfoque sistémico, a partir del reconocimiento de las posibilidades que ofrece el territorio en donde se ubican las zonas y/o áreas metropolitanas de los municipios centrales ${ }^{6}$ en proceso de consolidación en el Estado de Jalisco, México, y de los municipios exteriores que se agrupan en torno a estos municipios centrales conformando las zonas metropolitanas. Las ciudades medias elegidas para este caso de estudio son en la Región Ciénega del estado, donde se ubica la ciudad de Ocotlán (municipios centrales), Jalisco, México, esto por su peculiaridad en cuanto a su vocación turística-industrial y la otra en la Región Sur, donde se ubica; Ciudad Guzmán (municipios centrales), Jalisco, México, por el proceso de impacto negativo que se está teniendo en su territorio a través la agro-industrialización, que en origen esta ciudad se encuentra en una cuenca endorreica, con vocación forestal, de servicios y educación.

Por todo lo anteriormente expuesto se hace necesario tomar como punto de partida el asentamiento humano de mayor concentración "Municipio Central declaradas Zonas y/o Áreas Metropolitanas", como polo de desarrollo regional, (en estos dos caso de estudio, dentro de un corredor de traslado de mercancías nacional), a través del impacto que genera en el territorio (regional), partiendo del análisis de los aspectos ecológicos, ambientales, sociales económicos, políticos y culturales que se pretenden observar. Tomando en cuenta principalmente las vulnerabilidades naturales y antropogénicas que aquejan a estas zonas y/o áreas metropolitanas en su territorio, así como las repercusiones que están teniendo en el cambio climático y calentamiento global sobre la sustentabilidad.

Según la ONU-HÁBITAT (2016), las ciudades se dividen en cuatro categorías; las ciudades pequeñas que van de 100,000 a 500,000 habitantes, las ciudades intermedias que se consideran de 500,000 a 1,000,000 habitantes y otras dos categorías que no nos sirven para este estudio porque son de mayor población siendo solo la zona metropolitana de Guadalajara la que estaría en ese rango en el Estado de Jalisco. Las ciudades pequeñas e intermedias son consideradas según datos de los organismos como el (Banco de Desarrollo de América Latina (CAF), 2018) como los

\footnotetext{
${ }^{1}$ Centro de Investigación del Medio Ambiente y Ordenación del Territorio, UdG, https://orcid.org/0000-0002-6914-1288;

${ }^{2}$ Centro de Investigación Lago de Zapotlán y Cuencas, CUSur, UdG, https://orcid.org/0000-0002-7017-8360;

${ }^{3}$ Centro de Investigación Lago de Zapotlán y Cuencas, CUSur, UdG, https://orcid.org/0000-0001-9539-6623;

${ }^{4}$ Centro de Investigación Lago de Zapotlán y Cuencas, CUSur, UdG, https://orcid.org/0000-0002-6600-654X;

${ }^{5}$ Centro de Investigación Lago de Zapotlán y Cuencas, CUSur, UdG, https://orcid.org/0000-0002-7153-5980. * Correo de contacto: orendaintome@yahoo.com.mx.

${ }^{6}$ SEDATU, CONAPO e INEGI (2018), Delimitación de las zonas metropolitanas de México 2015, México.
} 


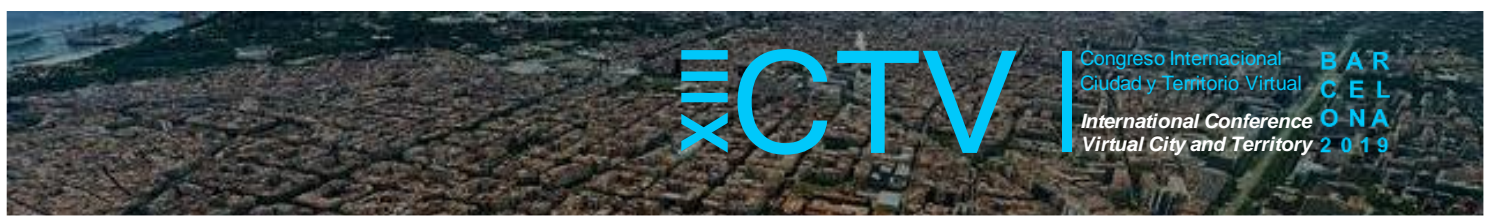

escenarios del desarrollo sustentable, es decir, son las ciudades intermedias las que podrían hacer un cambio de paradigmático del desarrollo urbanos que busca la descentralización, en el ordenamiento territorial de la región.

En el caso de nuestro país México las ciudades intermedias (rango de ONU-HÁBITAT (2016), juegan un papel fundamental, solo que, para el caso de estudio de las Ciudades Medias en el Estado de Jalisco, México, declaradas como zonas metropolitanas en nuestra legislación mexicana (SEDATU 2013), están en el rango de ciudades pequeñas según ONU-HÁBITAT (2016), puesto que ninguna rebasa el rango de los 500,000 habitantes.

De las dos ciudades medias incluidas en la Delimitación de las zonas metropolitanas de México 2015, (delimitación Federal oficial de CONAPO, INEGI y SEDATU); La zona metropolitana de Ocotlán, conformada por los municipios de Jamay, Ocotlán y Poncitlán, cuentan con una población de 176,158 habitantes, (censo de INEGI 2015), falta el decreto por el Congreso del Estado de Jalisco. En el caso del área metropolitana del sur, conformada por los municipios de Gómez Farías, Zapotiltic y Zapotlán el Grande del estado de Jalisco, cuenta con una población de 148,893 habitantes, (censo de INEGI 2015), esta área metropolitana solo fue decretada en el congreso del Estado de Jalisco, México y no está incluida en la delimitación de las zonas metropolitanas de México, (delimitación Federal oficial de CONAPO, INEGI y SEDATU).

\section{Abstract}

The Middle Cities in the process of consolidation as Metropolitan areas in Jalisco, generate positive expectations as an alternative of "Sustainable Development", and the decentralization of the Metropolitan area of Guadalajara, Jalisco, Mexico. This study will focus on two zones and/or metropolitan areas being the metropolitan areas of Ocotlán, in the Ciénega Region of the State of Jalisco, Mexico and the metropolitan area of the South of Jalisco, in the South Region of the State, in addition to the implementation of instruments such as the development and/or updating of the metropolitan program through the Territorial Planning in order to seek equity for Sustainable Urban Development that seeks impact in a more balanced way in its regions.

This research has a systemic approach, based om the recognition of the possibilities offered by the territory where the zones and/or metropolitan areas of the central municipalities are located in the consolidation process in the State of Jalisco, Mexico, and the outer municipalities which are grouped around these central municipalities forming the metropolitan areas. The average cities chosen for this case study are in the Ciénega region of the state, where the city of Ocotlán is located (central municipalities), Jalisco, Mexico, this due to its peculiarity in terms of its tourist-industrial vocation and the other in the South Region, where it is located; Ciudad Guzmán (central municipalities), Jalisco, Mexico, due to the negative impact process that is taking place in its territory through agro-industrialization, which in the beginning this city is located in an endorheic basin, with vocation for forestry, services and education.

For all the above, it is necessary to take as a starting point the highest concentration human settlement "Central Municipality declared Zones and/or Metropolitan Areas", as a regional development hub, (in these two case studies, within a corridor of national merchandise transfer), through the impact it generates in the (regional) territory, based on the analysis of the ecological, environmental, social, economic, political and cultural aspects that are intended to be observed. Taking into account mainly the natural and anthropogenic vulnerabilities that afflict these areas and / or metropolitan areas in their territory, as well as the impact they are having on climate change and global warming on sustainability.

According to ONU-HABITAT (2016), cities are divided into four categories; the small cities that range from 100,000 to 500,000 inhabitants, the intermediate cities that are considered from 500,000 to 1,000,000 inhabitants and two other categories that are not useful for this study because they are of greater population being only the metropolitan area of Guadalajara which would be in that rank in the State of Jalisco. Small and intermediate cities are considered according to data from organizations such as the (Development Bank of Latin America (CAF), 2018) as the scenarios of sustainable development, that is, it is the intermediate cities that could make a paradigm shift of the urban development that seeks decentralization, in the territorial planning of the region.

In the case of our country Mexico, the intermediate cities (ONU-HABITAT range (2016), play a fundamental role, only in the case of the study of the Medium Cities in the State of Jalisco, Mexico, declared as metropolitan areas in our Mexican legislation (SEDATU 2013), they are in the range of small cities according to UN-HABITAT (2016), since none exceeds the range of 500,000 inhabitants.

Of the two middle cities included in the Delimitation of the metropolitan areas of Mexico 2015, (official Federal delimitation of CONAPO, INEGI and SEDATU); The metropolitan area of Ocotlán, consisting of the municipalities of Jamay, Ocotlán and Poncitlán, has a population of 176,158 inhabitants, (census of INEGI 2015), the decree by the 


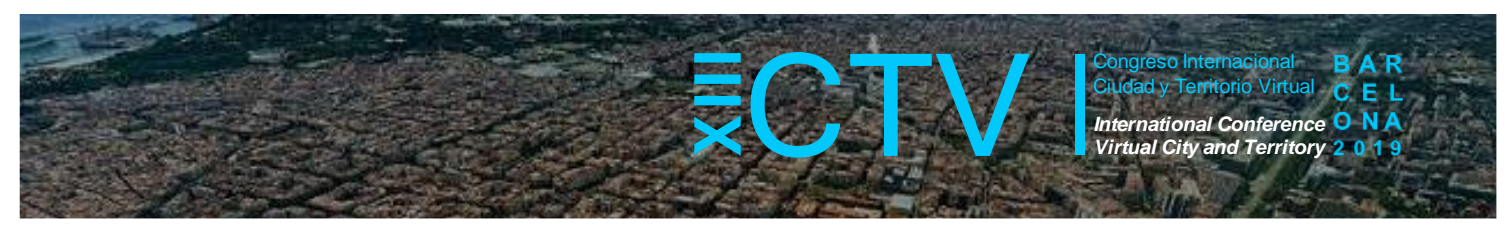

Jalisco State Congress is missing. In the case of the southern metropolitan area, formed by the municipalities of Gómez Farías, Zapotiltic and Zapotlán el Grande of the state of Jalisco, it has a population of 148,893 inhabitants, (census of INEGI 2015), this metropolitan area was only decreed in the congress of the State of Jalisco, Mexico and is not included in the delimitation of the metropolitan areas of Mexico, (official Federal delimitation of CONAPO, INEGI and SEDATU).

Palabras clave: Ciudades Medias; Metropolización; Sostenibilidad y Territorio

Key words: Middle Cities; Metropolis; Sustainability and Territory

\section{Introducción}

El presente documento de investigación presenta una aproximación para el análisis de dos Ciudades Medias, que fueron declaradas como zonas y/o áreas Metropolitanas en el Estado de Jalisco, México, que no han logrado cohesionarse o consolidarse como tal, sin embargo tanto la zona metropolitana de "Ocotlán", como el área metropolitana del "Sur", que son los casos de estudio, concentran las actividades socioeconómicas más representativas de la regiones "Ciénega y Sur", respectivamente del Estado de Jalisco, México; al concentrar el mayor numero de población en sus asentamientos humanos, y por consiguiente el equipamiento, infraestructura y servicios de mayor rango (estatal y Federal), para sus regiones.

La presente investigación tendrá un enfoque sistémico, a partir del reconocimiento de su ubicación en la cuenca hidrográfica, en la que se encuentra entendidas esta como el "territorio definido naturalmente, donde todos los procesos sociológicos están íntimamente ligados entre si... la posibilidad de realizar un manejo adecuado de su cuenca. Esto sucede cuando nos reconocemos como habitantes de ella y por lo tanto como beneficiarios de sus servicios eco sistémicos", SEMARNAT (2013)

\section{Objetivos}

\subsection{Objetivo General}

Se hace necesario tomar como punto de partida el asentamiento humano de mayor concentración de población (ciudad media y/o municipios centrales contenida en la declaración de área metropolitana), como polo de desarrollo, (en estos dos casos de estudio, mismos que están dentro de dos corredores de traslado de mercancías en el ámbito nacional e internacional, dentro de nuestro país México), a través del impacto que generan en el territorio, partiendo del análisis de los aspectos ecológicos, ambientales, sociales, económicos, políticos y culturales que se pretenden observar.

Tomando en cuenta principalmente las vulnerabilidades naturales y antropogénicas que aquejan a las zonas y/o áreas metropolitanas de sus principales asentamientos humanos en su territorio entendido este como la cuenca hidrográfica, para su subsistencia y su impacto ante el cambio climático y calentamiento global, sobre la sustentabilidad. Partiendo de los siguientes objetivos específicos como son el estudio de los siguientes elementos que integran su territorio:

- Análisis (hidrográfico-hidrológico) de los diferentes sistemas jerárquicos de cuencas hidrográficas, (cuencas. sub-cuencas y micro-cuencas)

\footnotetext{
7 SEMARNAT. (2013). Cuencas hidrográficas. Fundamentos y perspectivas para su manejo y gestión. Ciudad de México: Secretaría de Medio Ambiente y Recursos Naturales.
} 


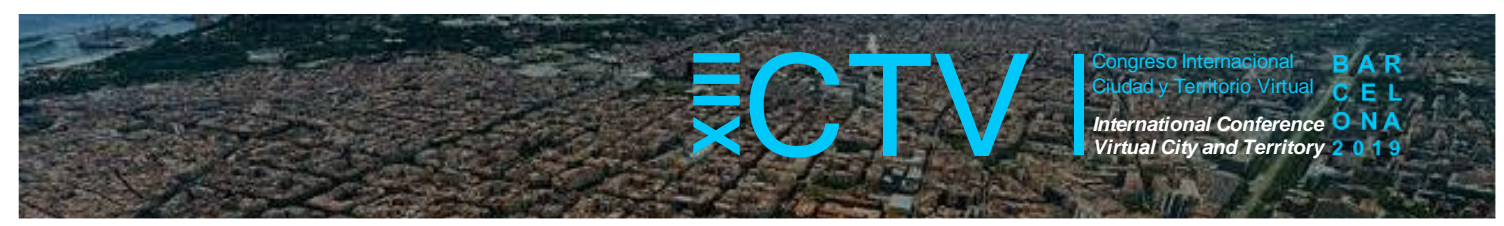

- Análisis del Ecosistema y de su interaccción con los ecosistemas naturales y/o inducidos en el entorno inmediato.

- Análisis de biodiversidad (centros de concentración de biodiversidad afectados por los asentamientos humanos y requerimientos para su preservación)

- Aspectos socio-ambientales

- Interacciones en su territorio; cambio de uso del suelo, cambio de la orografía a través de factores articifiales (presas, plataformación del territorio, ecurrimientos entre los más comunes), etc...

Por otra parte esta investigación gestionara para cada zona y/o áreas metropolitana el estudio y anális de los riesgos naturales a los que están expuestas las ciudades que se integran (municipio central y exterior en sus asentamientos humanos), y establecera el nivel de resiliencia, que deberá de estar enmarcada en las condiciones actuales, como lo señala la Ley General de Asentamientos Humanos y Ordenamiento Territorial ${ }^{8}$ (Congreso de la Unión, INFDM, CONEVAL, (2016) ${ }^{9}$, CONAPO, 2015 INEGI, 2016). Considerando el escenario máximo probable de intensidad y afectación (EMPIA), por lo que hay que construir los escenarios de intensidad máxima probable (IMP), para cada uno de los fenómenos perturbadores considerados para las diferentes zonas y/o áreas metropolitanas.

Las Ciudades Medias de esta investigación en proceso de consolidación como áreas Metropolitanas en Jalisco, México, generan expectativas positivas como una alternativa de "Desarrollo Sustentable", y descentralización del área Metropolitana de Guadalajara, Jalisco, México. Este estudio se centrará en dos zonas y/o áreas metropolitanas siendo las zonas metropolitanas de Ocotlán, en la Región Ciénega y área Metropolitana del Sur de Jalisco, en la Región Sur del Estado de Jalisco, México.

Se deben implementar los instrumentos normativos del ámbito territorial que establezca objetivos, estrategias y programas para el desarrollo sostenible de la zona y/o áreas metropolitanas en el corto plazo (5 años), mediano plazo (15 años) y largo plazo (30 años), en la elaboración y/o actualización de sus programas Metropolitanos a través del Ordenamiento Territorial en aras de buscar la equidad para el Desarrollo Urbano Sustentable que busque impactar de una manera más equilibrada, tomando como prioridad la preservación y restauración ecológico, el aprovechamiento sustentable de los recursos naturales y la protección al ambiente, y al medio socio-económico-cultural.

\section{Metodología}

Esta investigación tiene un enfoque sistémico, a partir del reconocimiento de las posibilidades que ofrece el territorio en donde se ubican las zonas y/o áreas metropolitanas de los municipios centrales en proceso de consolidación en el Estado de Jalisco, México, y los municipios exteriores que se agrupan en torno a estos municipios centrales conformando las zonas metropolitanas.

Las ciudades medias elegidas para esta investigación son "Ocotlán", en la Región Ciénega del estado de Jalisco, México, clasificada como municipio central, (SEDATU infografía figura No.1),

\footnotetext{
${ }^{8}$ Cámara de Diputados del H. Congreso de la Unión. (2016) Ley General de Asentamientos Humanos, Ordenamiento Territorial y Desarrollo Urbano. Ciudad de México.

${ }^{9}$ CONEVAL (2016). Medición de la pobreza 2010-2015. Consejo Nacional de Evaluación de la Política de Desarrollo Social. Ciudad de México: CONEVAL. Obtenido de Consejo Nacional de Evaluación de la Política de Desarrollo Social.
} 


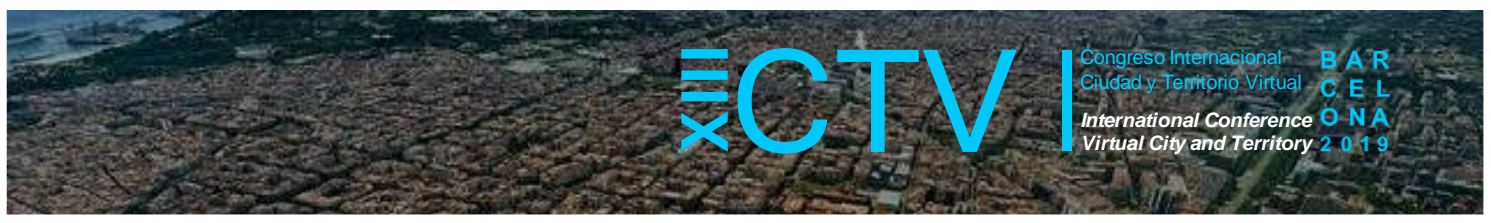

esto por su peculiaridad en cuanto a su vocación turística-industrial y la otra ciudad media es; "Ciudad Guzmán" en la Región Sur, del estado de Jalisco, México, clasificada también como municipio central, (SEDATU infografía figura No.1), y por el proceso de impacto negativo que se esta teniendo en su territorio a través la agro-industrialización (agricultura tecnificada siembra de aguacate y agricultura protegida invernaderos para producir berrys), que se ubica en la cuenca endorreica de la Laguna de Zapotlán, siendo esto una de sus máximas debilidades, además de que esta perdiendo su vocación forestal en la cuenca alta al experimentar un cambio de usos del suelo muy agresivo. Es por esto que este análisis será sistemático haciendo un análisis ecológico delimitado a partir de una lectura de su territorio, entendido este como su cuenca hidrográfica.

Figura 1. Infografía del método de Delimitación de las zonas metropolitanas de México 2015

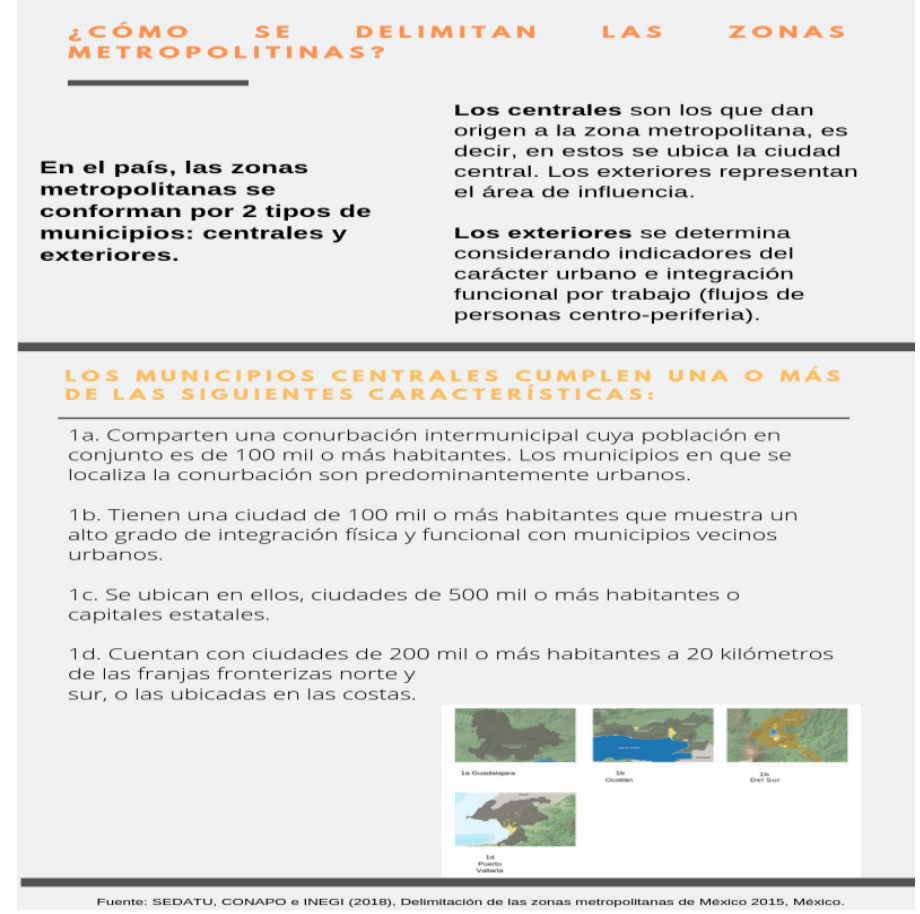

Por todo lo anteriormente expuesto se hace necesario tomar como punto de partida el asentamiento humano de mayor concentración "Municipios Centrales declarados como zonas y/o áreas metropolitanas", además de ser el polo de desarrollo regional, esto genera un impacto positivo desde el punto de vista económico y negativo desde el punto de vista eco sistémico en el territorio (región hidrográfica), partiendo del análisis de los aspectos ecológicos, ambientales, sociales económicos, políticos y culturales que se pretenden abordar. Tomando en cuenta principalmente las vulnerabilidades naturales y antropogénicas que aquejan a estas zonas y/o áreas metropolitanas en su territorio, así como las repercusiones que están teniendo en el cambio climático y calentamiento global sobre la sustentabilidad. Las ciudades medias metropolitanas en Jalisco, México hoy en día se han convertido en un factor determinante del desarrollo global-local de las regiones y a la vez en territorio sin directrices, pues el impacto de cambio de usos del suelo del Territorio y Urbano no se tenia previsto, acrecentar el impacto hacia el medio ambiente y lo único que importa es la creación de empleos o el desarrollo económico de estos dos polos de desarrollo emergentes "Municipios Centrales declarados zonas y/o áreas metropolitanas”. Según la ONU-HÁBITAT (2016), las ciudades se dividen en 


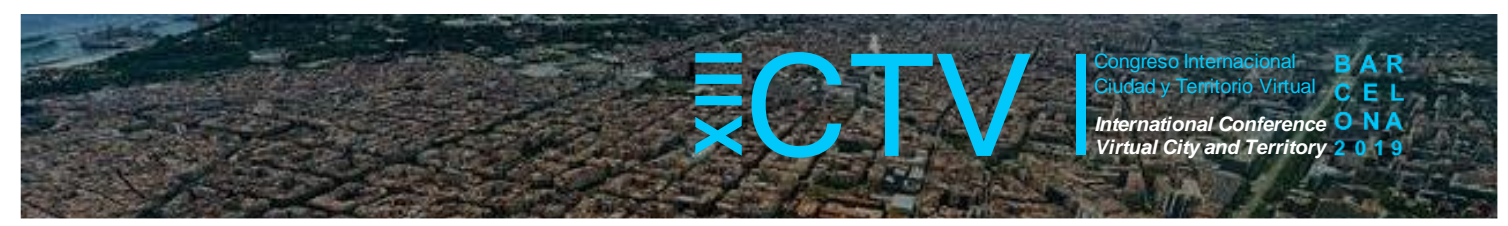

cuatro categorías; las ciudades pequeñas que van de 100,000 a 500,000 habitantes, las ciudades intermedias que se consideran de 500,000 a 1,000,000 habitantes y otras dos categorías que no nos sirven para este estudio por que son de mayor población siendo solo la zona metropolitana de Guadalajara la que estaría en ese rango. Las ciudades pequeñas e intermedias son consideradas según datos de los organismos como el (Banco de Desarrollo de América Latina $\left.{ }^{10}(C A F), 2018\right)$, como los escenarios del desarrollo sustentable, es decir, son las ciudades intermedias las que podrían hacer un cambio de paradigmático del desarrollo urbanos que busca la descentralización, en el ordenamiento territorial de la región.

En el caso de nuestro país México las ciudades intermedias (rango de ONU-HÁBITAT (2016), juegan un papel fundamental en la urbanización siendo solo el área metropolitana de Guadalajara conformada por 10 municipios la que cumple el rango de Ciudad grande; contabilizan entre 1 y 5 millones de habitantes, el área metropolitana de Guadalajara cuenta con una población de 4, 887,383 habitantes (INEGI 2015), solo que, para el caso de estudio de las Ciudades Medias en el Estado de Jalisco, México. Las Ciudades Medias declaradas como zonas metropolitanas en nuestra legislación Mexicana (SEDATU 2013) ${ }^{11}$, están en el rango de ciudades pequeñas según ONU-HÁBITAT (2016), puesto que ninguna rebasa el rango del los 500,000 habitantes. De las dos ciudades medias incluidas en la Delimitación de las zonas metropolitanas de México 2015 ${ }^{12}$, (delimitación Federal oficial de CONAPO, INEGI y SEDATU); solo aparece la zona metropolitana inter-estatal de Pto. Vallarta, conformada por los municipios de Pto. Vallarta, Jalisco, México y Bahía de Banderas, Nayarit, México, que cuenta con una población de 425,890 habitantes (censo de INEGI 2015), que no esta considerada en esta investigación.

\subsection{Casos de Estudio}

Las zona metropolitana de "Ocotlán", conformada por los municipios de Jamay, Ocotlán y Poncitlán, fue declarada en 2004, por SEDESOL, INEGI y CONAPO, como zona metropolitana, cuentan con una población de 176,158 habitantes, (censo de INEGI 2015), esta zona metropolitana si esta considerada en la Delimitación de las zonas metropolitanas de México 2015, delimitación Federal oficial de CONAPO, INEGI y SEDATU, (SEDATU infografía figura No.2), solo falta el decreto por el Congreso del Estado de Jalisco, para que sea reconocida a nivel estatal (tramite que se encuentra en proceso).

En el caso del área metropolitana del "Sur", conformada por los municipios de Gómez Farías, Zapotlán el Grande y Zapotíltic, fue declarada en 2012, por decreto del congreso del Estado de Jalisco, México, cuenta con una población de 148,893 habitantes, (censo de INEGI 2015), no está incluida en la Delimitación de las zonas metropolitanas de México 2015, delimitación Federal oficial de CONAPO, INEGI y SEDATU, (SEDATU infografía figura No.3). Está en trámite su integración al padrón de actualización de la delimitación Federal oficial de CONAPO, INEGI y SEDATU (2018), su proceso se encuentra en el Congreso de la Unión (Nivel Federal)

\footnotetext{
${ }^{10}$ Banco de Desarrollo de América Latina (CAF). (2018). Ciudades Medias. Trampolín para el desarrollo sostenible. Recuperado en marzo de 2019, de Banco de Desarrollo de América Latina: https://www.caf.com/es/conocimiento/blog/2018/08/ciudades-intermedias-trampolin-del-desarrollo-sostenible/

${ }_{11}^{11}$ SEDATU. (2013). Programa Nacional de Desarrollo Urbano y Ordenamiento del Territorio. México: Secretaria de Desarrollo Agrario, Territorial y Urbano.

${ }^{12}$ SEDATU. (2018). Delimitación de las zonas metropolitanas de México 2015, (SEDATU, INEGI y CONAPO). Primera edición en febrero 2018.
} 


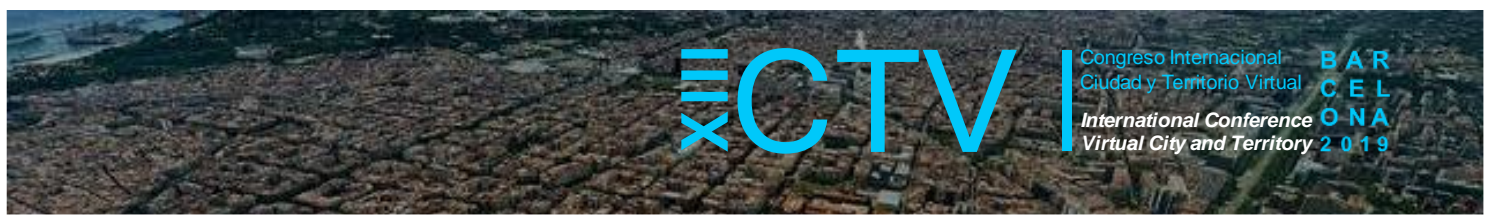

Figura 2. Infografía de la Delimitación de las zonas metropolitana de Ocotlán, Jalisco, México

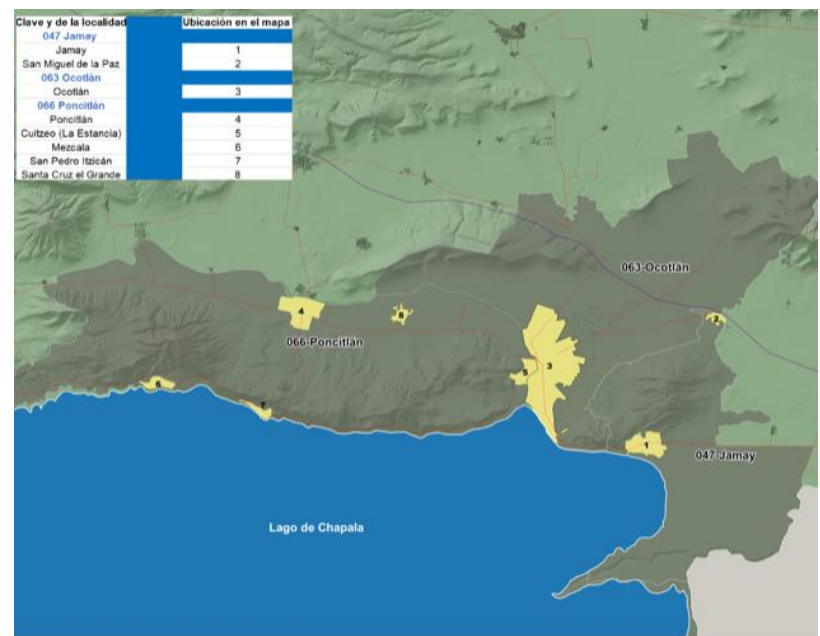

Fuente: SEDATU, INEGI, CONAPO 2015

Figura 3. Infografía de la Delimitación de las zonas metropolitana de Sur, Jalisco, México

Fuente: en base a los criterios de SEDATU, INEGI, CONAPO, sin estar en el padrón federal.

\subsection{Generalidades de las zonas y/o áreas metropolitanas}

La concentración de la población en zonas y/o áreas metropolitanas, trae como consecuencia aspectos positivos como negativos. En los positivo cabe destacar que en estas ciudades medias metropolitanas se concentran las actividades: industrias, agroindustriales, los servicios de carácter regional y esto por consiguiente genera la inversión en los dos casos de estudio. Su dinamismo es tal, que hoy en día son los motores del crecimiento económico, al generar el $75 \%$ del Producto Interno Bruto (PIB), (SEDESOL, CONAPO, INEGI, 2007). Por estas razones, las zonas y/o áreas metropolitanas siguen atrayendo a la población rural, y se refuerza lo que siempre ha sido una constante en la política territorial mexicana, fortalecer o atender a las ciudades.

En cuanto a las problemáticas que presentan las zonas y/o áreas metropolitanas, (Iracheta, 2010), identifica los siguientes elementos: 


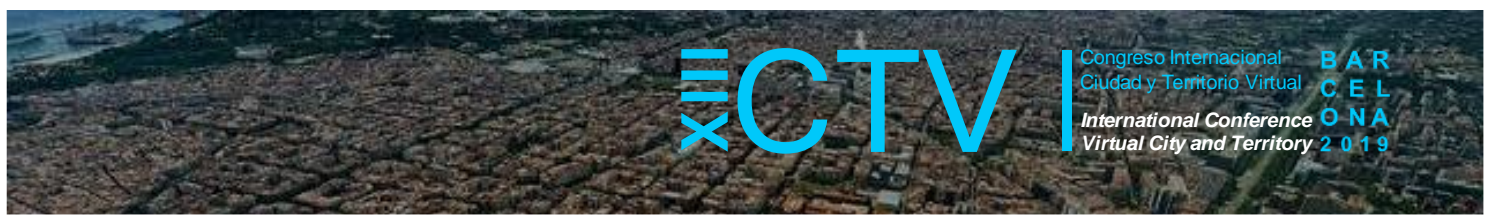

a) tienen un patrón de crecimiento urbano disperso;

b) sus periferias presentan enormes rezagos en la dotación de servicios e infraestructura;

c) carecen de un adecuado marco legal e institucional de coordinación intergubernamental, entre los tres niveles de gobierno (Municipal, Estatal y Federal).

De lo anterior, llama la atención que desde el punto de vista político-institucional centrándose en la toma de decisiones políticas no existe un marco jurídico claro que regule el desarrollo urbano del país y el mecanismo para gestionar las zonas metropolitanas sea solo la buena voluntad de los gobiernos municipales en turno para su coordinación (Arellano, 2013 y 2014). Un avance significativo ha sido, sin duda, la aparición en 2006 de los Fondos Metropolitanos asignados a través de Ramo 23 "Provisiones Salariales". Éstos aparecieron como un mecanismo para resolver algunas problemáticas urbanas, aunque sus Reglas de Operación aparecieron dos años después. Al no tomarse en consideración algunas peculiaridades que guardan las zonas metropolitanas del país en cuanto a su rango o tamaño se vuelve complicada la coordinación entre los órdenes de gobierno del federal, estatal y municipal, para aplicar las "Reglas de Operación". Los problemas de coordinación se dificultan en zonas metropolitanas con las características siguientes: 1) las de gran tamaño en donde confluyen una enorme constelación de actores e instituciones, un ejemplo es la zona metropolitana del Guadalajara y 2) que el país cuenta con zonas metropolitanas de carácter interestatal el caso de la zona metropolitana de Puerto Vallarta que implica al municipio de Puerto Vallarta del Estado de Jalisco, México y el municipio de Bahía de Banderas del Estado de Nayarit, México.

Por lo anterior y debido a cuestiones de tipo normativo, los Fondos Metropolitanos sufrieron modificaciones en 2012 y 2014. También se debe mencionar que en este periodo de tiempo se han incrementado los montos, como el número de zonas metropolitanas favorecidas con dichos recursos. En 2006 y 2007 la zona metropolitana del Valle de México fue la única beneficiada, para 2008 el monto de los Fondos Metropolitanos se destinó a seis zonas metropolitanas; y en 2013 los recursos de esta partida presupuestal se canalizaron a 47 zonas metropolitanas (Iracheta, 2014). En el cuadro 4, se puede ver el incremento de los montos en los Fondos Metropolitanos a partir de la creación de este fondo de procedencia federal, mismo que solo se puede acceder si el área esta considerada en la Delimitación de las zonas metropolitanas de México 2015, delimitación Federal oficial de CONAPO, INEGI y SEDATU, (2018).

Tabla 1. Montos de los Fondos Metropolitanos (2006-2014)

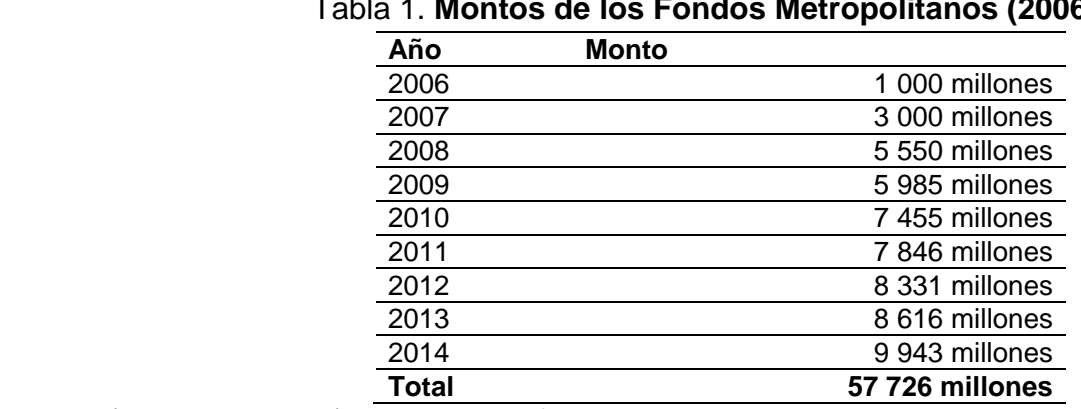

Fuente: (Moreno, 2010: 18) y Presupuesto federal- Ministrado.

De ahí que el aporte económico se da por primera vez para las dos zonas metropolitanas, no tan representativas como son "Ocotlán (vocación industrial) y Puerto Vallarta (vocación turística) " del Estado de Jalisco México ciudades medias del interior del estado de Jalisco, México, la gestionan de recursos de los Fondos Metropolitanos se generan hasta el 2014, para obras de infraestructura vial y de movilidad motorizada y no motorizada en estas dos zonas 


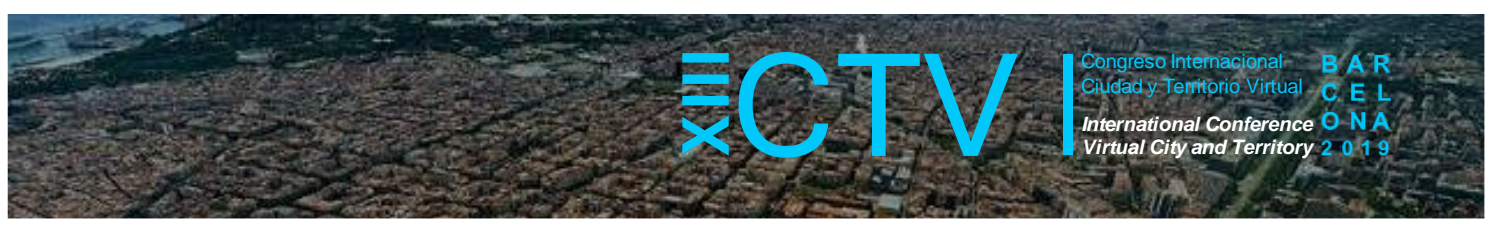

metropolitanas, pero que en una visión de conjunto son de gran interés e importantes para la descentralización del Estado de Jalisco, México.

\section{Marco jurídico de los fondos Metropolitanos}

Las Reglas de Operación establecen tres cuerpos colegiados:

1.- Consejo para el Desarrollo Metropolitano (CODEM);

2.- Comité Técnico del Fideicomiso del Fondo Metropolitano y;

3.- Subcomité Técnico de Evaluación de Proyectos.

El Consejo para el Desarrollo Metropolitano (CODEM), tiene como atribuciones:

a) definir los objetivos, prioridades, políticas y estrategias para el desarrollo de cada zona metropolitana;

b) determinar los criterios para la alineación de los planes, estudios, evaluaciones, acciones, programas, proyectos y obras de infraestructura y su equipamiento que se postulen al Fondo Metropolitano;

c) aprobar la cartera de programas y proyectos de inversión que someta a su consideración el Subcomité Técnico de Evaluación de Proyectos, así como las modificaciones que pudieran requerirse en tanto se acredite su pertinencia y contribución al desarrollo de la zona metropolitana correspondiente; y

d) remitir trimestralmente el informe del destino y aplicación de los recursos, del avance físico y financiero y de la evaluación de los resultados alcanzados y el impacto urbano, económico y social al Subcomité Técnico de Evaluación de Proyectos, así como a la Comisión para el Desarrollo Metropolitano de la Cámara de Diputados.

Por su parte, el Comité Técnico del Fideicomiso del Fondo Metropolitano tiene como competencias:

a) autorizar la entrega de recursos con cargo al patrimonio del fideicomiso, previo análisis y recomendación favorable del Subcomité Técnico de Evaluación de Proyectos;

b) dar seguimiento al avance financiero y físico de los estudios, planes, evaluaciones, programas, proyectos, acciones, obras de infraestructura y su equipamiento apoyados y definir las evaluaciones de sus resultados; $y$

c) autorizar con cargo a los recursos del fideicomiso, el uso de los rendimientos financieros y ahorros derivados de los procesos de contratación de las obras (Reglas de Operación del Fondo Metropolitano, 2014).

Mientras que el Subcomité Técnico de Evaluación de Proyectos tiene las funciones de:

a) apoyar al Comité Técnico en el análisis de las evaluaciones de impacto metropolitano, regional, económico, social y ambiental, así como en los análisis costo y beneficio de los estudios, planes, evaluaciones, programas, proyectos, acciones, obras de infraestructura y su equipamiento;

b) emitir recomendaciones para la autorización de recursos; y

c) dar seguimiento al avance físico y financiero de los estudios, planes, evaluaciones, programas, proyectos, acciones, obras de infraestructura (Reglas de Operación del Fondo Metropolitano, 2014) ${ }^{13}$.

13 Reglas de Operación del Fondo Metropolitano, 2014, Diario Oficial de la Federación, en http:// www.dof.gob.mx/nota_detalle.php?codigo53 34584\&fecha=04/03/2014, consultado el 25 de agosto de 2014 . 


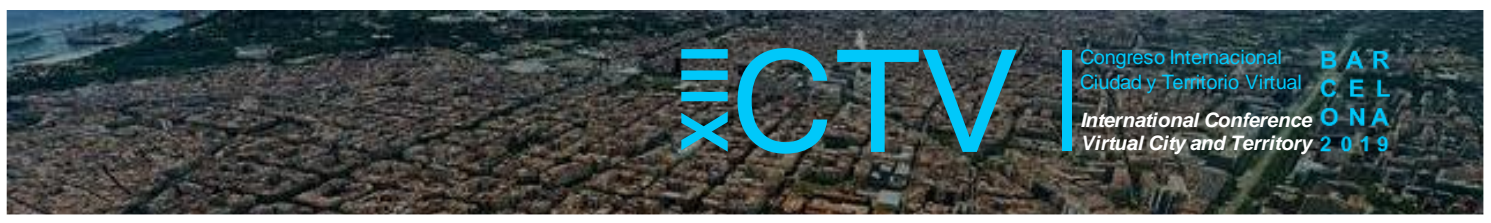

\section{La aplicación de los fondos metropolitanos en la ciudad media de Ocotlán, Jalisco}

Respecto a la zona metropolitana de Ocotlán se debe mencionar que ésta fue delimitada por SEDESOL, CONAPO y INEGI, desde el año de 2004. La zona metropolitana de "Ocotlán", se ubica en la región de la Ciénega en los márgenes del Lago de Chapala. Las instancias que establecen la delimitación antes mencionadas, consideraron que la zona metropolitana de Ocotlán se integrara por el municipio central que dio origen a la conurbación "Ocotlán", junto con los municipios de Poncitlán y Jamay como municipios exteriores jerarquización que se establecen para la determinación de la zona metropolitana de Ocotlán, cuenta con una población de 176,153 habitantes (encuesta inter-censal 2015 de INEGI), cuya tasa de crecimiento medio anual es $1.5 \%$ y se asienta en una superficie de $1240.3 \mathrm{~km} 2^{14}$.

No obstante, y pese a que las instancias de planeación reconocieran a la zona metropolitana de "Ocotlán", como tal, tuvieron que pasar seis años para que se declarara oficial y jurídicamente su existencia. ${ }^{15} \mathrm{~A}$ partir del caso de Ocotlán se debe notar que el interés para la creación y delimitación de la zona metropolitana Ocotlán, como seguramente ocurre en todo México, es para acceder a los Fondos Metropolitanos como recursos financieros para obras de infraestructura que deberían ser de impacto metropolitano. Por dicha razón, en 2010 las autoridades de los ayuntamientos de Ocotlán, Poncitlán y Jamay enviaron la propuesta de decreto de zona metropolitana al Congreso del Estado misma que fue decretada hasta este año 2019. Llama la atención la inclusión del municipio de Jamay que en origen no estaba contemplada en la zona metropolitana, pero cabe advertir que en este proceso de conformación de la zona metropolitana no se escapa el protagonismo de la ciudad central que dio pie a su conformación, pues como lo expone Adriana Hernández: "A partir del decreto de la zona metropolitana de Ocotlán se ha iniciado una nueva reorganización territorial, lo que lleva a una tendencia de privilegiar los procesos urbanos e industriales en la ciudad central: Ocotlán" (2013: 112) ${ }^{16}$.

\subsection{Organismos con los debe de operar una zona y/o área metropolitana}

En el caso de Ocotlán se conforma, el Consejo Metropolitano de Ocotlán, Poncitlán y Jamay en año 2011, a partir de esta fecha comenzaron a cristalizarse obras derivadas del Fondo Metropolitano de Jalisco, México administrado por el Gobierno del Estado, en su ejecución de obras por la Secretaria de Infraestructuras y Obras Públicas del Estado (SIOP), la primera obra pública ejecutada de este fondo fue el parque lineal o ciclovía que une a los tres municipios mediante una zona que cruce desde la carretera de Poncitlán a Jamay con una ciclo vía y bancas de descanso para disfrutar el paisaje puesto que en la mayor parte esta va bordeando el Lago de Chapala (Hernández, 2013: 107-108). Desde entonces, y durante el periodo 20112014, la Zona Metropolitana de Ocotlán ha recibido 114.7 millones de pesos, de los cuales el $46 \%$ se destinó a la construcción o mantenimiento de obras viales principalmente en el

\footnotetext{
14 Fuente: Elaborado por el Grupo Interinstitucional con base en la Encuesta Intercensal 2015, declaratorias y programas de ordenación de zonas conurbadas y zonas metropolitanas.

${ }_{15}$ Al respecto la legisladora perredista, Olga Araceli Gómez Flores, presidenta de la Comisión de Asuntos Metropolitanos, mencionó que "Esto requería una validez real, ya que en este caso la CONAPO, desde hace varios años los reconoce como zonas metropolitanas, administrativamente, no así a nivel de decreto, lo cual implica un incremento, en este caso sería el presupuesto de los ayuntamientos y que, además, tiene que ver con obras ya etiquetadas" (El Informador, 7 de abril de 2010).

16 Espacio Público, núm. 47, septiembre--diciembre 2016, pp. 77--98.
} 


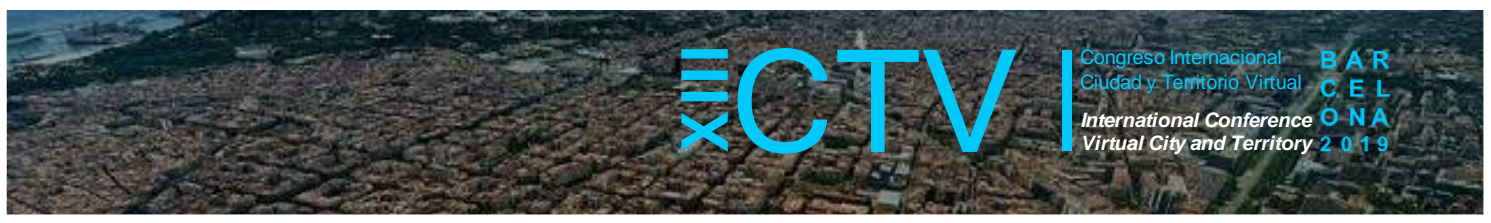

Municipio central de Ocotlán, el $29 \%$ a movilidad no motorizada (ciclovía citada) y el $25 \%$ a espacios públicos dispersos y sin rango de impacto metropolitano. Cabe precisar que para el 2014 la zona metropolitana de Ocotlán, tenia una población de 164, 256 habitantes, mientras que su parque vehicular fue 55, 482 automotores, lo que significa que por cada 2.9 habitantes existe un vehículo (INEGI, 2015), en el caso de los fondos metropolitanos uno de los factores importantes dentro de las convocatorias de apoyo por parte de la federación (SEDATU 2019), para las zonas metropolitanas es incidir en estas zonas para des-centivar el uso del automóvil.

En el caso del área metropolitana del sur esta se decreta en el congreso local, hasta el año de 2012 y es en el año 2017 cuando se sube al congresos de la unión, para que se reconozca como cómo zona metropolitana y se pueda reconocer en la SEDATU ${ }^{17}$, Delimitación de las zonas metropolitanas de México 2018, México. Secretaría de Desarrollo Agrario, Territorial y Urbano. Misma que por los tiempos políticos y cambio de gobierno federal quedo pendiente y hasta la fecha no se ha actualizado este padrón, por lo cual esta área metropolitana ha quedado incompleta en cuanto a su consolidación motivo por el cual no le es posible acceder a los fondos metropolitanos, además de que no se han puesto de acuerdo los municipios que conforman esta área metropolitana crear los organismos que establece la Ley General de Asentamientos Humanos y Ordenamiento Territorial.

\section{Reflexiones finales}

En el análisis de los Fondos Metropolitanos se puede sostener que son el principal instrumento de la política del gobierno federal en materia metropolitana; en tanto que para los municipios son el principal incentivo para coordinarse y hacer la obra pública, aunque en muchas ocasiones no es de impacto metropolitano que por su cuenta difícilmente pueden construir. Los Fondos Metropolitanos nacen en 2006 como una respuesta para atender principalmente la infraestructura metropolitana.

Al realizar el análisis se constató que son un mecanismo que continuamente se rediseña porque desde su origen no se tomaron en cuenta algunas de las peculiaridades que guardan algunas zonas metropolitanas del país que no entran en los esquemas tradicionales, tales como: el tamaño de algunas de ellas como en nuestro caso de estudio el municipio central es por lo menos en población tres veces mayor que sus municipios externos. Además, se debe reconocer que, por su diseño organizativo, la toma de las decisiones del destino de los Fondos Metropolitanos no es democrático. Esto es, que la participación ciudadana y voto de otros actores que ayudarían a la conformación de contrapesos a la hora de tomar decisiones sobre dichos recursos es nula. No obstante, la gestión para la obtención de dichos montos obliga a los gobiernos locales a coordinarse y sumar esfuerzos, aunque en la práctica, el ejercicio y control de los mismos es el gobierno del estado al ser el encargado de la edificación de las obras públicas de infraestructuras que principalmente se han destinado a la movilidad motorizada en el caso de Jalisco, México.

Del ejercicio de los Fondos Metropolitanos en las áreas urbanas estudiadas de Jalisco, y durante el periodo 2006-2014, se observa que las zonas metropolitanas han recibido un total de $7115.1 \mathrm{mdp}$, lo que significa el $12.3 \%$ del total del fondo a nivel nacional. La zona metropolitana más beneficiada ha sido la de Guadalajara con 6780.2

17 SEDATU, CONAPO e INEGI (2018), Delimitación de las zonas metropolitanas de México 2015, México. Secretaría de Desarrollo Agrario, Territorial y Urbano. 


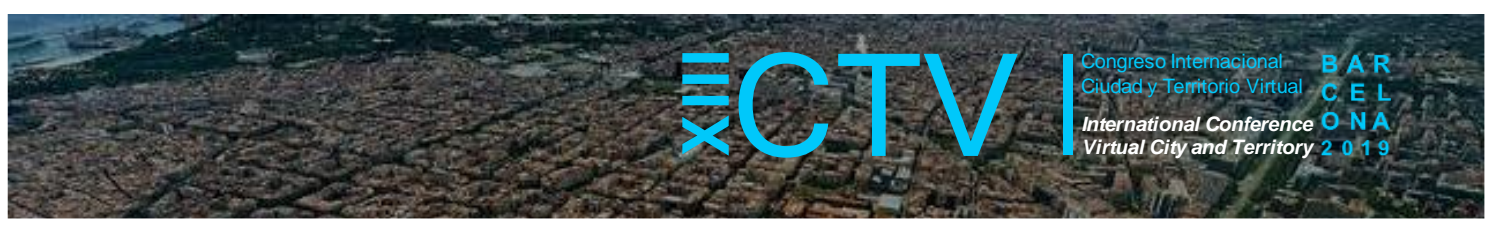

millones de pesos, lo que representa el 11.74\%; muy por debajo le siguió la zona metropolitana de Puerto Vallarta con 220.2 millones de pesos, es decir 0.38 por ciento y finalmente la zona metropolitana de Ocotlán con 114.7 millones de pesos., que representa $0.19 \%$.

De lo anterior, se deduce que la mayoría de los recursos de los Fondos Metropolitanos se aplicaron a obras viales, a dicho rubro, para Ocotlán el $46 \%$. El segundo rubro fue la movilidad no motorizada ciclo vías que une a los tres municipios, el $29 \%$ para Ocotlán. El tercer rubro fue para la rehabilitación de los espacios públicos, en Ocotlán $25 \%$, y finalmente, llama la atención que ante la diversificación de los montos de los Fondos Metropolitanos que realizó la zona metropolitana de Ocotlán esto fue en el periodo de 2011-2014, la zona metropolitana de Guadalajara empezó a diversificarlos hasta 2013 con el destino de recursos a la rehabilitación de espacios públicos y la construcción de ciclo vías. Sin embargo, un aspecto negativo para el caso de Ocotlán es que dichos recursos favorecen el protagonismo económico de la ciudad central.

Se espera la diversificación de los Fondos Metropolitanos a través de la Ley de Coordinación Metropolitana con la constitución de cuatro instancias: la Junta de Coordinación Metropolitana, el Instituto Metropolitano de Planeación, el Consejo Consultivo de Planeación Metropolitana y el Consejo Ciudadano Metropolitano. El nuevo entramado institucional busca que la coordinación metropolitana beneficie a la población en general de cada área metropolitana; y que en las obras de movilidad motorizada y no motorizada (transporte público masivo y alternativo o alimentador), se desincentive el uso del automóvil, y la infraestructura metropolitana (principalmente de asegurar el recursos hídricos y tratamiento sustentable de los desechos solidos desde una visión metropolitana). Por otro lado, el consenso político y discurso económico global proyectan que se debe planear y construir zonas metropolitanas competitivas sustentables, pero también socialmente responsables con el medio ambiente e inclusivas.

Contribuciones de los autores: El primer autor ha desarrollado los puntos 2,4,5 y 6 , el segundo autor ha el punto 1,3 y 6 , el tercer autor los puntos 3 y 6 , etc. El segundo y tercer autor participaron en los puntos que se coincide en los apartados que se repiten en numero.

Conflicto de Intereses: Los autores declaran que no hay conflicto de intereses.

\section{Bibliografía:}

Arellano Ríos, A. (2013), La gestión metropolitana. Casos y experiencias de diseño institucional, México: unam-iij-El Colegio de Jalisco.

Arellano Ríos, A. (2014), "La condición política de la coordinación metropolitana en Guadalajara, México", en Revista Estado, Gobierno y Gestión Pública, vol. 24, núm. 3, pp. 89120.

Cámara de Diputados del H. Congreso de la Unión. (2016) Ley General de Asentamientos Humanos, Ordenamiento Territorial y Desarrollo Urbano. Ciudad de México. 


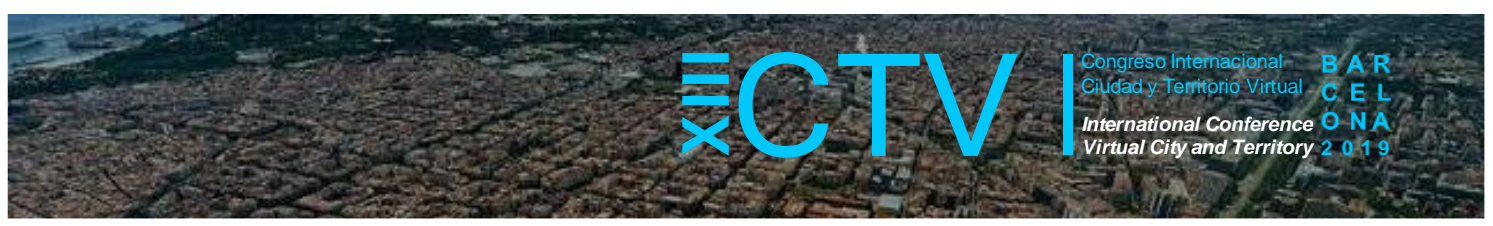

Cárdenas Gómez, E \& Arellano Ríos, A (2016), Los Fondos Metropolitanos en tres áreas urbanas: Guadalajara, Ocotlán y Puerto Vallarta, Espacio Público. ISSN 1665-8140, Electrónico: en trámite, núm. 47, septiembre-diciembre 2016, pp. 77-98

CONEVAL (2016). Medición de la pobreza 2010-2015. Consejo Nacional de Evaluación de la Política de Desarrollo Social. Ciudad de México: CONEVAL. Obtenido de Consejo Nacional de Evaluación de la Política de Desarrollo Social.

Iracheta, A. (2010), "El fenómeno metropolitano en México", en Economía y Sociedad, vol. xiv, núm.25, pp.153-179.

Iracheta, A. y J. Iracheta (2014), Evaluación de los Fondos Metropolitano y Regional del Gobierno Federal Mexicano, México: cide- Centro clear para América Latina, shcp-bid- Colegio Mexiquense-Centro Eure, México.

Hernández García, A. (2013), "La zona metropolitana de Ocotlán: políticas públicas y actores sociales en su conformación", en A. Arellano e I. Ortiz (coords.). Coordinación y gestión metropolitana en Jalisco, Puerto Vallarta: CuCosta-Universidad de Guadalajara, pp. 93-118.

Moreno, S. (2010), Los resultados de los fondos metropolitanos en México, México: Centro de Estudios Sociales y de Opinión Pública-Cámara de Diputados, Documento de Trabajo, núm.87. SEDATU. (2013). Programa Nacional de Desarrollo Urbano y Ordenamiento del Territorio. México: Secretaria de Desarrollo Agrario, Territorial y Urbano.

SEDATU, CONAPO e INEGI (2018), Delimitación de las zonas metropolitanas de México 2015, México. Secretaría de Desarrollo Agrario, Territorial y Urbano.

SEMARNAT. (2013). Cuencas hidrográficas. Fundamentos y perspectivas para su manejo y gestión. Ciudad de México: Secretaría de Medio Ambiente y Recursos Naturales.

Reglas de Operación del Fondo Metropolitano, 2014, Diario Oficial de la Federación. Disponible en http://www.dof.gob.mx/nota_detalle.php?codigo53 34584\&fecha=04/03/2014 